\title{
PRESENCIA DE LENGUAJE ACADÉMICO EN LAS EDUCADORAS DE PÁRVULOS Y SU RELACIÓN CON EL DESARROLLO DEL LENGUAJE DE LOS NIÑOS $^{1}$
}

\author{
Ernesto Treviño, Carla Varela, Francisca Romo y \\ Valeska Núñez ${ }^{2}$
}

\begin{abstract}
RESUMEN
La investigación indaga en la relación entre el lenguaje que utilizan las educadoras de párvulos en sus interacciones con los estudiantes y los resultados de lenguaje de estos últimos. Específicamente, se analiza la complejidad sintáctica, la diversidad léxica y la presencia de palabras sofisticadas en el discurso de las educadoras, en breves experiencias de aprendizaje en las que las profesoras tienden a tomar más la palabra, así como utilizar el lenguaje para transmitir conocimiento. Los resultados indican que, controlando por variables socioculturales del niño y variables de la sala de clases, como la calidad de las interacciones y el cambio de educadora entre prekínder y kínder, existen relaciones significativas de las variables de diversidad léxica y uso de palabras sofisticadas de parte de las educadoras en la identificación de letras y palabras, y en la escritura emergente de los niños. Se discute la importancia del lenguaje de la educadora para disminuir la brecha entre el lenguaje de contextos familiares de los niños y el requerido en la escuela.
\end{abstract}

Palabras clave: lenguaje académico, lenguaje oral de los profesores, discurso pedagógico, interacciones de aula, desarrollo lingüístico.

\section{PRESENCE OF ACADEMIC LANGUAGE IN PRE-SCHOOL EDUCATORS AND ITS RELATION TO STUDENTS' LANGUAGE DEVELOPMENT}

\section{ABSTRACT}

This article analyzes the relationship between pre-school teachers' language use in classrooms and students' language development. Specifically, this piece studies syntactic complexity, lexical diversity and the presence of sophisticated words in teachers' speech, in activities in which teachers tend to lead the conversation and to use language to transmit knowledge. The results indicate that controlling for socio-cultural variables of the child and variables of the classroom, such as the quality of the interactions and changing teachers from pre-kindergarten to kindergarten, the variables of the teacher's lexical diversity and use of sophisticated words significantly predict children's results on the identification of letters and words and emergent writing. Finally, this text discusses the importance of teacher language in reducing the gap between the language used in family contexts and the language required in school.

Keywords: academic language, oral teacher language, pedagogical discourse, classroom interactions, language development.

1 El presente estudio ha sido financiado por el Consejo Nacional de Educación, en el marco de la convocatoria de apoyo a la investigación en temas de educación 2014. Los autores agradecen a Ignacio Wyman y Felipe Godoy, investigadores del Centro de Políticas Comparadas de Educación, por su apoyo en los análisis estadísticos de la presente investigación.

2 Todos los autores pertenecen al Centro de Políticas Comparadas de Educación, Universidad Diego Portales, Santiago, Chile. Contacto: ernesto.trevino@mail.udp.cl 
138 PRESENCIA DE LENGUAJE ACADÉMICO EN LAS EDUCADORAS DE PÁRVULOS Y SU RELACIÓN CON EL DESARROLLO DEL LENGUAJE DE LOS NIÑOS - E. Treviño, C. Varela, F. Romo y V. Núñez

\section{Introducción}

Los niños y niñas de nivel socioeconómico bajo tienen en la escuela su principal vía para acercarse al lenguaje formal que se usa en los contextos escolares. El presente estudio se ha propuesto determinar la relevancia del uso de lenguaje académico por parte de las educadoras de párvulos, en los resultados de aprendizaje de lenguaje de los niños a los que enseñan. La literatura indica que el uso de este registro por parte de los adultos que enseñan a los niños, predeciría su comprensión lectora y su desarrollo lingüístico (Dickinson, Hofer, Barnes y Grifenhagen, 2014; Harper-Browne y Raikes, 2012; Ravid y Tolchinsky, 2002). Para ello se ha descrito el lenguaje académico utilizado por las educadoras de párvulos, de prekínder y kínder, en sus interacciones de aula con los niños y se ha relacionado su uso con los resultados en lenguaje de estos.

\section{Marco teórico}

En este estudio, el lenguaje es entendido desde una perspectiva sociolingüística, a partir de los postulados de la Lingüística Sistémico Funcional (M. A. K. Halliday, 1979) y la Sociología del Discurso en la teoría de Basil Bernstein $(1985,1993)$. El discurso pedagógico, en este contexto, se refiere una construcción social que se realiza en el marco de las interacciones que se dan en las salas de clase. En este sentido, las interacciones, que se realizan o no con la ayuda del lenguaje, surgen y están determinadas por su contexto social. Específicamente, y siguiendo los planteamiento de Deborah Hicks (1996), el discurso del aula escolar implica, como cualquier otro discurso, una forma determinada y relacionada con unas prácticas comunicativas sociales. El discurso de la sala, así entendido, es un marco de conocimiento previo que permite que los profesores y los niños se comuniquen y construyan sus propios significados dentro de su contexto y su actividad compartida. Para ello, estos participantes utilizan unos géneros definidos en el espacio de la escuela (el momento de contar el cuento, las definiciones, las descripciones, exposiciones, etc.). Cada género podría tener su propio registro -los rasgos léxicos y gramaticales que caracterizan un uso del lenguaje-, pero Schleppegrell (2001) muestra que 
estos géneros compartirían unos rasgos comunes, puesto que responderían a propósitos similares.

Con todo, este discurso, en el caso de la sala de clases, se construye a partir de las interacciones entre niños y adultos. Estas interacciones se entienden, en la presente investigación, desde lo propuesto por Pianta, La Paro y Hamre (2008) en el marco de su instrumento de observación de aula CLASS (Classroom Assessment Scoring System). Se trata del intercambio comunicativo entre los estudiantes y el profesor (o adulto a cargo) en una sala de clases ${ }^{3}$.

\subsection{Interacciones en el aula}

El lenguaje académico se realiza, entre otros, en el contexto de las interacciones comunicativas. En la sala de clases, estas interacciones se generan entre el profesor y sus estudiantes, o entre los estudiantes mismos. El instrumento CLASS (Pianta et al., 2008) propone un lente para observar la efectividad ${ }^{4}$ de estas interacciones.

Este instrumento supone que las interacciones de aula pueden agruparse en dominios (y estos, a su vez, en dimensiones), los cuales en su conjunto resumen los principales atributos de una enseñanza efectiva. En específico, el CLASS propone que las interacciones de calidad se centran en tres dominios: a) apoyo socioemocional; b) organización de la sala; y, c) apoyo instruccional. Un buen profesor sería aquel que crea un clima de respeto y confianza para expresar dudas y no temer a los errores. Este profesor es capaz de mantener una organización de la clase que permite un ritmo intenso de aprendizaje, con actividades encadenadas y transiciones efectivas y breves entre ellas, al mismo tiempo que moviliza el desarrollo conceptual y lingüístico de los estudiantes, porque entrega retroalimentación individualizada a cada cual, lo que le permite expandir sus ideas y profundizar su aprendizaje. Así, un docente aprovecha las intervenciones de los niños y niñas para proponer nuevas preguntas, problemas, ideas,

3 Este instrumento es explicado en el marco metodológico del estudio.

4 Se entiende que las interacciones efectivas son aquellas que, por un lado, generan un contexto lo más adecuado posible para el aprendizaje, y, por otro, entregan las mayores oportunidades para que los estudiantes aprendan. 
140 PRESENCIA DE LENGUAJE ACADÉMICO EN LAS EDUCADORAS DE PÁRVULOS Y SU RELACIÓN CON EL DESARROLLO DEL LENGUAJE DE LOS NIÑOS - E. Treviño, C. Varela, F. Romo y V. Núñez

que motiven una conversación extendida, desafiante cognitivamente, que amplíe las oportunidades de aprendizaje. Asimismo, dentro del dominio apoyo pedagógico, se consideran aspectos referidos a la calidad y cantidad de técnicas de facilitación lingüística, junto con el uso del lenguaje de el o la educadora como modelo de habla (Pianta et al., 2008). Considerados en conjunto, estos dominios han probado ser un buen indicador de las oportunidades de aprendizaje y un predictor de los resultados de desempeño en estudios de educación preescolar y primaria (Stuhlman y Pianta, 2009).

En un marco de didáctica general, el enfoque situado resulta coherente con propuestas como la de Vygotsky $(1934,2002)$ que permiten comprender los procesos de enseñanza y de aprendizaje como interacciones verbales entre adultos y niños o, más específicamente, entre un sujeto más preparado y un aprendiz. En efecto, así entendida la interacción en el aula, reconoce que el aprendizaje no se produce por una transferencia automática de conceptos de un sujeto a otro, sino que a través de la mediación que proporcionan las personas, las herramientas y, principalmente, el lenguaje, en el contexto de situaciones definidas social y culturalmente.

Variadas investigaciones en las salas de Educación de Párvulos en Chile (Concha, Treviño, Romo, Varela, Arratia, Garrido y Arias, 2010; Lagos, Treviño, Concha, Romo y Varela, 2010; Treviño, Toledo y Gempp, 2013; Leyva, Weiland, Barata, Yoshikawa, Snow, Treviño y Rolla, 2015; Yoshikawa, Leyva, Snow, Treviño, Barata, Weiland y Arbour, 2015) muestran que las interacciones que se observan en estas aulas poseen mejores resultados en los dominios que se relacionan con lo socioemocional y organizacional, por sobre las dimensiones que están directamente relacionadas con el aprendizaje de los estudiantes: desarrollo de conceptos, calidad de la retroalimentación y modelaje lingüístico.

\subsection{La lingüística sistémico funcional y la sala de clases}

Dentro de las corrientes lingüísticas, la sistémico funcional (LSF) ha dedicado gran parte de su tiempo al estudio del lenguaje dentro de la sala de clases. Se trata de un modelo principalmente gramatical que describe el lenguaje en cuanto se configura como un recurso para 
construir e interpretar significados en contextos sociales (Halliday, 1979). En este sentido, los usos del lenguaje se construyen desde los contextos sociales, incluidas las personas que participan de ellos (tomando en cuenta su educación, clase social, relaciones de poder, etc.), los lugares, propósitos, etc.

Los investigadores de este modelo que se han dedicado a estudiar el lenguaje de la escuela, sobre todo en los niveles mayores (Christie y Martin, 2007; Martin, 2013; Moyano, 2013; Nagy y Townsend, 2012; Schleppegrell, 2012; Snow, 2010). Los estudios se han enfocado en describir algunos recursos lingüísticos que se utilizan para construir conocimientos (sobre todo en las ciencias, con el ejemplo de las metáforas) para explorar la construcción de conocimientos lingüísticos al aprender una segunda lengua; o para especificar las características propias del habla de la sala de clases y la escritura en el contexto educativo.

Así, la LSF ha descrito un registro que sería propio de la sala de clases: el lenguaje académico. Por registro, se entiende la variación de la lengua, de acuerdo con lo que una persona está haciendo. Se trata de:

[...] la configuración de recursos semánticos que un miembro de una cultura asocia de manera típica con el tipo de situación [...]. Tanto la situación como el registro asociado a ella pueden describirse con diferentes grados de especificidad; pero la existencia de registros es una evidencia de la experiencia cotidiana: los hablantes no tienen dificultad en reconocer las opciones y combinaciones semánticas que están en juego en determinadas condiciones contextuales. Dado que estas opciones son realizadas bajo la forma de la gramática y el vocabulario, el registro puede reconocerse como una selección particular de palabras y estructuras, pero se define en términos de significado [...] (Halliday, 1979, p. 145-146).

Bajo esta perspectiva, entonces, los estudiantes que participan del contexto situacional de la sala de clases deberían poseer oportunidades para conocer este registro que los inscribe en la cultura escolar. Esto, por supuesto, debería suceder desde que entran a la escuela. 
142 PRESENCIA DE LENGUAJE ACADÉMICO EN LAS EDUCADORAS DE PÁRVULOS Y SU RELACIÓN CON EL DESARROLLO DEL LENGUAJE DE LOS NIÑOS - E. Treviño, C. Varela, F. Romo y V. Núñez

\subsubsection{Registro: lenguaje académico en el aula}

Schleppegrell (2012) define el lenguaje académico como un registro (o conjunto de registros) a través del cual los niños aprenden. Ellos se encuentran por primera vez con este uso del lenguaje cuando llegan a la escuela. De este modo, los adultos presentes en la sala son los responsables de usarlo para que los estudiantes también puedan hacerlo. Este hecho posee mucha relevancia, puesto que el lenguaje académico es intrínsecamente más difícil que otros registros (dados los recursos lingüísticos que utiliza) y que el pensamiento que promueve su desarrollo es una tarea clave que deben trabajar todos los educadores con sus estudiantes (Snow y Ucceli, 2009).

Como cualquier registro lingüístico, el lenguaje académico es de naturaleza dinámica y multidimensional (Halliday, 1993; Snow, 2010) no hay solo un lenguaje académico, pues este depende de los participantes, el lugar, la cultura, el modo, etc. que lo configura a partir de sus propósitos. Con todo, todas las variedades de lenguaje académico poseen ciertos rasgos comunes.

En general, el lenguaje académico que ha recibido más atención es el escrito, y dentro de este, el que se utiliza para referirse a la ciencia. En este contexto, se trata de un conjunto de saberes y habilidades lingüísticas sofisticadas que permiten un pensamiento más complejo, como el que es propio del mundo académico (Snow y Ucceli, 2009). Sin embargo, la importancia y urgencia del lenguaje académico en el discurso oral de la sala de clases, de estudiantes de todas las edades, son claras (Dickinson et al., 2014). La literatura de interacciones educativas ha mostrado en variadas ocasiones que las habilidades que debiesen relevarse son las habilidades de pensamiento superior: metacognición, razonamiento, creatividad, etc. (Pianta et al., 2008).

Así, el lenguaje académico, como un registro abstracto que se expresa en otros registros específicos dependientes de los factores del contexto de situación, se define desde uno de los extremos de un continuum. En este se encuentra el lenguaje académico, por un lado, y el lenguaje conversacional e informal, por el otro. En el extremo del lenguaje académico más formal, escrito la mayor parte de las veces, algunos de los rasgos lingüísticos que se identifican son: 
mayor concisión, gran densidad de información (expresada en el uso de palabras más densas semánticamente), precisión, etc. Para el lenguaje más conversacional se observa un mayor uso de oraciones que comienzan con pronombres o sujetos animados, verbos que se refieren a acciones más que a relaciones, y oraciones largas que poseen información secuenciada (Snow, 2010). El lenguaje oral (más conversacional) de la sala de clases (más académico) poseería características de ambos extremos.

\subsubsection{Algunos rasgos del lenguaje académico de la sala de clases}

En la presente propuesta, se consideran algunos rasgos lingüísticos específicos para el lenguaje académico, observados por (Dickinson et al., 2014) en su estudio en algunas salas de Head Start ${ }^{5}$ :

a. Características estructurales: una sintaxis más compleja y un vocabulario preciso que se relaciona con los contenidos que tienden a ser más infrecuentes. Específicamente, se trata de oraciones largas (mayor número de palabras) y complejas (que poseen una o varias cláusulas) (Huttenlocher, Vasilyeva, Cymerman y Levine, 2002). Asimismo, en el caso del vocabulario se observa el uso de palabras más sofisticadas (palabras que se presentan con menor frecuencia en los registros conversacionales, informales) y una mayor variedad léxica (Dickinson y Porche, 2011).

Respecto del vocabulario sofisticado, Schleppegrell (2012) reconoce que la presencia de palabras propias de lo que se está enseñando, es decir, la terminología de la disciplina (en el caso, por ejemplo, de la enseñanza del lenguaje, términos como "palabras", "autor", "letras", etc.) es una característica del lenguaje académico. El uso de esta nomenclatura ayudaría a los estudiantes a participar de tareas cognitivas más complejas y hacer elecciones discursivas efectivas.

b. Características funcionales: el lenguaje académico debería presentarse en aquellos contextos donde hay un propósito conceptual y reflexivo, discusiones que expanden y profundizan el conocimiento de mundo de los niños. Se trataría, entonces,

5 Programa estadounidense que se hace cargo de la preparación de niños más vulnerables para la entrada a la escuela. 
de conversaciones conceptuales (conceptual talk) acerca de temas provenientes de la ciencia, la historia, la lectura (por ejemplo, conversaciones respecto del significado de determinadas palabras).

\section{Marco metodológico del estudio}

\subsection{Muestra de salas}

Se utilizaron videos recolectados en el contexto de la evaluación del Programa "Un Buen Comienzo" (UBC) ${ }^{6}$ en su modelo de estudio experimental durante los años 2008 y 2011. Específicamente, se analizaron los videos para tres tiempos (antes y al final de prekínder, y al final de kínder) de las salas del grupo control de la segunda cohorte del estudio (2009-2010).

De este modo, la muestra quedó conformada por 14 salas, cada una con tres videos $(n=42)$, de la segunda cohorte del grupo control del estudio experimental "Un Buen Comienzo" (escuelas municipales de Maipú y Lo Prado). Estas aulas, que cuentan con las mismas características demográficas que las del grupo de intervención ${ }^{7}$, solo recibieron algunos materiales (libros) de parte de la fundación que implementaba el proyecto. Es decir, este grupo posee condiciones business-as-usual.

Estos videos registran una jornada diaria completa de trabajo en aula, de aproximadamente cuatro horas. En general, estas salas cuentan con una educadora a cargo y una técnico o asistente de párvulos. Además, poseen 30 niños en promedio.

\subsection{Muestra de los niños}

Como se ha dicho, este estudio se relaciona directamente con los datos recolectados en el marco de la evaluación del proyecto "Un Buen

6 Programa de desarrollo profesional docente, implementado por la Fundación Educacional Oportunidad, que capacita y entrega coaching en la sala a educadoras y técnicos en ejercicio de prekínder y kínder, por dos años.

7 Las escuelas municipales de estas comunas (como las escuelas a las que pertenecen las salas del estudio) poseen un número de estudiantes prioritarios en los niveles de transición de un $30 \%$ o más. 
Comienzo", en su diseño experimental. En este contexto, se considera una muestra total de 289 niños que pertenecen a las salas analizadas.

Las variables consideradas para los niños son expuestas más adelante, en el apartado metodológico del análisis final.

\subsection{Procedimientos de análisis}

\subsubsection{Codificación 1: Modalidades del discurso pedagógico}

Para seleccionar las actividades de cada uno de los videos, se decidió observar las jornadas completas (los videos completos) considerando un criterio discursivo que permitiera amplitud en la revisión pero que, a la vez, entregara un criterio más preciso de qué segmentos podían ser más representativos del uso del lenguaje (ampliamente) del adulto a cargo de la sala, y dentro de estos segmentos, de la presencia de un registro específico en las interacciones verbales: el lenguaje académico.

El concepto utilizado para la revisión de las jornadas de párvulos es el de discurso pedagógico (Bernstein, 1993), que se operacionaliza en modalidades. El énfasis del concepto de discurso pedagógico, en la teoría de Bernstein está puesto en cómo sirve para la recontextualización y reconstrucción de los conocimientos en la escuela. Este discurso podría regirse por dos tipos de reglas distintas, que relacionan y entrelazan el conocimiento y el discurso en la sala:

- El discurso instruccional, que regula la transmisión de competencias y habilidades especializadas. En este sentido, se trataría sobre todo de la relación entre discursos primarios más especializados y específicos a áreas del conocimiento y el discurso en la sala de clases.

- El discurso regulativo, que regula la forma en que se construye un orden y una relación e identidad social. Específicamente, se trata de la relación entre un discurso social que dictaría reglas de identidad, principios de orden y de establecimiento de relaciones y el discurso en la sala de clases.

De este modo, en esta primera fase de codificación, codificadores previamente entrenados observaron y clasificaron cada minuto de 
las jornadas consideradas en la muestra (42 videos), asignando las siguientes clasificaciones ${ }^{8}$ :

- Discurso instruccional.

- Discurso regulativo.

- Sin discurso: no se producen interacciones verbales entre la educadora y los niños.

- No codificable: no se oye o entiende lo que la educadora dice, se canta o se lee un texto.

Para esta fase, el equipo de codificación obtuvo, en promedio, un $70 \%$ de concordancia entre sí. Esta medida resulta de la consideración de la cantidad de coincidencias en la asignación de una clasificación para cada uno de los minutos de los videos dobles codificados (20\% de la muestra). Además, una supervisora revisó, al azar, 20 minutos de todo el resto de videos. Este proceso permitió tomar acuerdos que facilitaron llegar al porcentaje de concordancia informado.

\subsubsection{Selección de experiencias de aprendizaje a transcribir para análisis}

A partir del proceso de codificación 1, se seleccionaron 10 minutos pertenecientes a dos experiencias de aprendizaje, para cada jornada de la muestra. La elección de fragmentos de 10 minutos para transcribir está alineada con las elecciones llevadas a cabo en otros estudios que dan cuenta de que es una medida confiable respecto de lo que sucede en una jornada (Dickinson y Porche, 2011; Dickinson et al., 2014). Esta se realizó siguiendo los siguientes criterios:

- Experiencias de aprendizaje que contengan al menos un $70 \%$ de minutos con discurso instruccional (es decir siete minutos del total, que era 10). Estos siete minutos con DI podían ser seguidos o estar intercalados con minutos que tuvieran otra clasificación.

8 El discurso que puede presentar la educadora en un minuto, por supuesto, podría considerar variabilidad en términos de la modalidad del discurso. En este sentido, la manera de codificar implicó, algunas veces, que el codificador tomara la decisión respecto de asignar DI o DR. Para no generar inconcordancias entre los codificadores, el criterio aplicado fue el siguiente: considerando los 60 segundos que se observan, si en más de 30 segundos se observa DI, asigne ese código (el mismo criterio se aplica para todas las otras clasificaciones). 
- Experiencias de aprendizaje que consideren una actividad variable con propósito pedagógico, priorizando aquellos objetivos menos frecuentes, como los relativos a Ciencia o Matemática, y una actividad no variable con propósito pedagógico (que resultó ser, en la mayoría de los casos, el momento de saludo, al principio de la jornada).

Es importante notar que estos criterios muestran el supuesto de la probabilidad de que hay mayor presencia de lenguaje académico cuando se usa discurso instruccional, lo que se basa en la conceptualización misma de esta modalidad de discurso. Además, se considera que en las actividades con propósito pedagógico, se encuentran más oportunidades de observar la modalidad de discurso instruccional.

Aunque los criterios a priori parecieron razonables para encontrar varias experiencias que cumplieran con ellos, este supuesto no se cumplió, sino que sirvió de manera justa para encontrar dos experiencias pedagógicas: una actividad variable y una no variable. En efecto, en algunos casos fue necesario hacer concesiones respecto del primer criterio, puesto que los minutos codificados como DI no abundaban.

De este modo, la muestra quedó constituida por 84 segmentos de actividad (dos por video). Estos tienen en promedio ocho minutos (con un rango de cinco a 12 minutos).

\subsubsection{Codificación 2: Análisis de experiencias pedagógicas con} CLASS

El estudio consideró el análisis de cada experiencia pedagógica con el instrumento de evaluación de interacciones efectivas CLASS, Classroom Assessment Scoring System (Pianta et al., 2008).

Para asegurar la calidad de este proceso de codificación, un 20\% de la muestra se codificó dos veces y se supervisó de cerca este proceso. Con estas medidas, se pudo mantener la confiabilidad de la codificación (Cohen's kappa > 0,80). 
2.3.4. Codificación 3: Transcripción de las experiencias pedagógicas con CHAT y análisis de lenguaje académico

Las experiencias de aprendizaje seleccionadas en el proceso $1 \mathrm{y}$ codificadas con el instrumento CLASS fueron transcritas utilizando el formato CHAT, del programa Child Language Data Exchange System, CHILDES (MacWhinney, 2000). Solo fue considerado el discurso del adulto, fuera educadora o asistente de párvulos. Un 20\% de las experiencias, seleccionadas aleatoriamente, fueron doblemente transcritas. La calidad de este proceso se analizó considerando la totalidad de coincidencias en la transcripción de una misma actividad sobre la totalidad de palabras transcritas ( $80 \%$ de coincidencias entre transcriptores). Además, al igual que en las anteriores etapas, una supervisora se hizo cargo de la calidad del proceso y de preguntas o dificultades que pudieran surgir en el mismo.

Las transcripciones producidas en esta etapa se analizaron utilizando el programa CLAN, el cual sirve para analizar variables lingüísticas presentes en el discurso del adulto. En específico, se realizó un análisis del lenguaje desde una perspectiva estructural, considerando:

- La diversidad de vocabulario usando el D (Durán, Malvern, Richards y Chipere, 2004), una medida de uso de palabras distintas un poco más sofisticada (basada en la probabilidad de aparición de vocabulario diferente en muestras de lo que se está analizando) que la razón de palabras distintas sobre total de palabras del discurso (o tokens).

- La complejidad sintáctica, usando la medida MLU (mean length of utterance). Esta medida indica la cantidad de palabras por utterance (unidad de sentido y de entonación) que utiliza, en promedio, un hablante en su discurso analizado.

- Presencia de vocabulario sofisticado. En el estudio de Dickinson et al. (2014), esta variable fue construida post hoc, es decir, a partir del corpus de palabras presentes en las transcripciones, se realizó un análisis de cuáles podían ser las más sofisticadas en comparación con la misma muestra. En el caso de la presente investigación, se realizó igualmente un análisis post hoc, considerando la totalidad de palabras presentes en la muestra. De esta muestra se eliminaron 
los nombres propios (de los participantes de la actividad en curso o de lugares u otro), onomatopeyas y algunas palabras propias de la sala, más dialectales (por ejemplo: pechocho). Las palabras restantes fueron clasificadas como sofisticadas (1) o no sofisticadas (0), tomando en consideración la lista de palabras comunes (que deben conocer los niños al terminar $4^{\circ}$ básico), según el programa de estudio de este nivel del Ministerio de Educación, Mineduc (2003). Se revisó otro listado de palabras frecuentes el que, sin embargo, estaba basado en un corpus periodístico y escrito que no aplicaba de igual manera al corpus. La medida de presencia de vocabulario sofisticado, finalmente, está compuesta por una razón (cuya medida va de 0 a 1) entre la presencia de palabras sofisticadas sobre el total de palabras observado para un hablante.

Para el análisis final, se consideraron solo las medidas de las educadoras de párvulos (y no las de las técnicos), por abarcar estas la mayor cantidad del tiempo observado en cada actividad.

2.4. Relación entre resultados de lenguaje de los estudiantes y presencia de lenguaje académico: variables utilizadas y proceso

Para el análisis final, se realizó una regresión que considera como variable de resultado cuatro medidas de lenguaje de los niños asistentes a las salas en cuestión. Estos resultados fueron recogidos utilizando el Woodcock-Muñoz Language Survey Revised (WMLS-R) (Woodcock, Muñoz, Ruef y Alvarado, 2005). En específico, se trata de cuatro pruebas:

- vocabulario sobre dibujos,

- identificación de letras y palabras,

- dictado,

- comprensión de textos.

De este modo, se realizaron dieciséis (16) modelos distintos, considerando cada prueba de los niños y, dentro de las variables explicativas, cada variable de lenguaje académico de las educadoras por separado, además de todas las variables de lenguaje académicas al mismo tiempo. 
Las variables que se consideran en el modelo de regresión son:

- Variables de resultado: 1. Puntaje en WM para vocabulario; 2. Puntaje en WM para identificación de letras y palabras; 3. Puntaje en WM para dictado; 4. Puntaje en WM para comprensión de textos.

- Variables explicativas: 1. Variables de lenguaje académico: a) complejidad sintáctica (MLU), b) diversidad de palabras (D), c) presencia de vocabulario sofisticado; 2 . Variables de calidad de interacciones en la sala: puntaje CLASS (promedio de las 10 dimensiones evaluadas); 3. Variable de cambio de educadora desde prekínder a kínder; 4. Variables socioeconómicas de los niños: para controlar por una variable que diera cuenta del contexto familiar del que provenía cada niño, se consideró el nivel educativo de la madre a) educación básica completa, b) educación media incompleta, c) educación media completa, d) estudios superiores; y 5. Variables demográficas del niño o niña:

a) su edad en meses y b) su género.

Además, en estos análisis se controló por los resultados de entrada de los niños de la muestra, es decir, los resultados obtenidos por los mismos niños al principio del año para cada prueba considerada.

\section{Resultados}

En la siguiente sección se exponen, en primer lugar, los resultados del análisis de la calidad de las interacciones en las experiencias pedagógicas consideradas. Luego, se da cuenta de los resultados para las variables de lenguaje académico. Finalmente, se muestran los análisis de regresión que dan cuenta de la relación existente entre los resultados de lenguaje de los niños y las variables consideradas en el estudio presente.

\subsection{Resultados de interacciones pedagógicas}

Para el análisis de las interacciones, se ha observado un total de 84 actividades (dos por cada uno de los 42 videos), con una duración promedio de 11,07 minutos. Un resumen de los resultados descriptivos para este análisis, se muestra en la siguiente tabla: 
Tabla 1: Descriptivos de las dimensiones del CLASS para las actividades observadas en los tres tiempos considerados

\begin{tabular}{l|c|c|c|c|c|c|c|c|c|c}
\hline & \multicolumn{3}{|c|}{ Tiempo } & \multicolumn{3}{c|}{ Tiempo 2 } & \multicolumn{3}{c}{ Tiempo 3 } \\
\cline { 2 - 11 } & Act. 1 & Act. 2 & Promedio & Act. 1 & Act. 2 & Promedio & Act. 1 & Act. 2 & Promedio \\
\hline Clima positivo & 5,07 & 4,50 & 4,79 & 4,73 & 4,53 & 4,63 & 4,86 & 4,21 & 4,54 \\
& $(0,62)$ & $(0,85)$ & $(0,79)$ & $(1,16)$ & $(0,99)$ & $(1,07)$ & $(1,29)$ & $(1,19)$ & $(1,26)$ \\
\hline Clima negativo* & 1,29 & 1,14 & 1,21 & 1,20 & 1,47 & 1,33 & 1,36 & 1,43 & 1,39 \\
& $(0,47)$ & $(0,36)$ & $(0,42)$ & $(0,56)$ & $(0,74)$ & $(0,66)$ & $(0,50)$ & $(0,76)$ & $(0,63)$ \\
\hline Sensibilidad de la & 5,29 & 4,86 & 5,07 & 5,00 & 4,73 & 4,87 & 4,29 & 4,71 & $4,50]$ \\
educadora & $(1,07)$ & $(0,86)$ & $(0,98)$ & $(1,13)$ & $(1,22)$ & $(1,17)$ & $(1,44)$ & $(1,44)$ & $(1,43)$ \\
\hline Consideración por & 3,14 & 2,86 & 3,00 & 4,07 & 3,27 & $3,67+$ & 3,57 & 3,50 & $3,54]$ \\
la perspectiva de & $(0,95)$ & $(1,03)$ & $(0,98)$ & $(1,33)$ & $(0,88)$ & $(1,18)$ & $(1,09)$ & $(0,85)$ & $(0,96)$ \\
los niños & 5,43 & 4,71 & 5,07 & 4,87 & 4,60 & 4,73 & 4,93 & 4,21 & $4,57]$ \\
\hline Manejo de la & $(1,09)$ & $(1,44)$ & $(1,30)$ & $(1,41)$ & $(1,30)$ & $(1,34)$ & $(1,38)$ & $(1,37)$ & $(1,40)$ \\
conducta & 5,86 & 5,79 & 5,82 & 5,33 & 5,40 & 5,37 & 5,64 & 5,43 & $5,54]$ \\
\hline Productividad & $(1,10)$ & $(1,12)$ & $(1,09)$ & $(1,05)$ & $(1,06)$ & $(1,03)$ & $(1,15)$ & $(1,28)$ & $(1,20)$ \\
\hline Formato para & 4,29 & 4,43 & 4,36 & 4,40 & 4,07 & 4,23 & 4,57 & 4,14 & $4,36]$ \\
aprender & $(0,73)$ & $(0,76)$ & $(0,73)$ & $(0,74)$ & $(0,80)$ & $(0,77)$ & $(0,94)$ & $(1,23)$ & $(1,10)$ \\
\hline Desarrollo de & 2,29 & 2,14 & 2,21 & 2,27 & 2,07 & 2,17 & 2,57 & 2,43 & $2,50]$ \\
conceptos & $(0,91)$ & $(0,77)$ & $(0,83)$ & $(0,88)$ & $(0,88)$ & $(0,87)$ & $(0,85)$ & $(1,16)$ & $(1,00)$ \\
\hline Calidad de la & 2,71 & 2,71 & 2,71 & 2,67 & 2,60 & 2,63 & 2,71 & 2,64 & $2,68]$ \\
retroalimentación & $(0,83)$ & $(0,83)$ & $(0,81)$ & $(0,98)$ & $(0,99)$ & $(0,96)$ & $(1,14)$ & $(1,28)$ & $(1,19)$ \\
\hline Modelaje lingüístico & 3,64 & 3,79 & 3,71 & 3,53 & 3,00 & 3,27 & 3,86 & 3,43 & $3,64]$ \\
& $(1,15)$ & $(1,12)$ & $(1,12)$ & $(1,30)$ & $(0,93)$ & $(1,14)$ & $(1,23)$ & $(1,74)$ & $(1,50)$ \\
\hline PROMEDI0S & 3,90 & 3,69 & 3,80 & 3,81 & 3,57 & 3,69 & 3,84 & 3,61 & $3,73]$ \\
& $(0,89)$ & $(0,91)$ & $(0,90)$ & $(1,05)$ & $(0,98)$ & $(1,02)$ & $(1,10)$ & $(1,23)$ & $(1,17)$ \\
\hline
\end{tabular}

${ }^{* * *} p<0,01 ; * * p<0,05 ;{ }^{*} p<0,1$

+El promedio para "Consideración por la perspectiva de los niños" es significativamente más alto para el tiempo 2, solo en relación con el tiempo 1.

Se han marcado en negritas las dimensiones que poseen el puntaje más alto y el más bajo para los tres tiempos.

* Esta dimensión es invertida, pues su medida es contraria a las medidas del resto de las dimensiones.

Como se observa, las salas analizadas no muestran variación significativa en los tres tiempos considerados respecto del puntaje total del CLASS (los puntajes van de 3,69 puntos a 3,8 puntos). Esto quiere decir que, en promedio, la calidad de las interacciones de las salas, en específico, las actividades consideradas para este análisis, se encuentra en un nivel medio.

Respecto de las dimensiones que poseen una mejor evaluación, destaca la de productividad, con un puntaje medio alto (en el caso de las actividades del tiempo 1, llega a estar más cerca del nivel alto). La dimensión de desarrollo de conceptos, por su parte, se muestra como 
la más descendida con puntajes que se consideran en el nivel bajo de la evaluación de las interacciones. Es notable el hecho de que la dimensión de modelaje lingüístico posea puntajes algo superiores a la dimensión consideración por la perspectiva de los niños: en general, en las investigaciones revisadas (Concha et al., 2010; Lagos et al., 2010; Treviño et al., 2013; Yoshikawa et al., 2015) se observan todas las dimensiones de apoyo pedagógico obteniendo menores resultados que las dimensiones de apoyo socioemocional y organización del aula. Ahora bien, esto podría deberse a la selección de las actividades observadas con el lente CLASS: experiencias de aprendizaje en las que la educadora tiende a tener la palabra y, consecuentemente, a dirigir la clase, y, aunque podría ser que se generen conversaciones con los niños, estas están determinadas por la planificación de la educadora (hablar respecto de la fecha, del clima, de la lectura que se está realizando, etc.).

Ahora bien, este instrumento agrupa las diez dimensiones arriba mostradas en tres dominios más generales. Los resultados para estos dominios provienen del promedio simple de las dimensiones que los componen, a saber: apoyo socioemocional (clima positivo, clima negativo, sensibilidad de la educadora y consideración por la perspectiva de los niños), organización del aula (manejo de la conducta, productividad y formatos para aprender), y apoyo instruccional (desarrollo de conceptos, calidad de la retroalimentación y modelaje lingüístico). En la siguiente tabla se observan los promedios para cada tiempo y actividad, para estos dominios. 
Tabla 2: Descriptivos de los dominios del CLASS para las actividades observadas en los tres tiempos considerados

\begin{tabular}{l|c|c|c|c|c|c|c|c|c|c}
\hline & \multicolumn{3}{|c|}{ Tiempo 1 } & \multicolumn{3}{c|}{ Tiempo 2 } & \multicolumn{3}{c}{ Tiempo 3 } \\
\cline { 2 - 10 } & Act. 1 & Act. 2 & Promedio & Act. 1 & Act. 2 & Promedio & Act. 1 & Act. 2 & Promedio \\
\hline $\begin{array}{l}\text { Apoyo } \\
\text { socioemocional }\end{array}$ & $\begin{array}{c}3,70 \\
(0,78)\end{array}$ & $\begin{array}{c}3,34 \\
(0,78)\end{array}$ & $\begin{array}{c}3,52 \\
(0,79)\end{array}$ & $\begin{array}{c}3,75 \\
(1,05)\end{array}$ & $\begin{array}{c}3,5 \\
(0,96)\end{array}$ & $\begin{array}{c}3,63 \\
(1,02)\end{array}$ & $\begin{array}{c}3,52 \\
(1,08)\end{array}$ & $\begin{array}{c}3,46 \\
(1,06)\end{array}$ & $\begin{array}{c}3,49 \\
(1,07)\end{array}$ \\
\hline $\begin{array}{l}\text { Organización } \\
\text { del aula }\end{array}$ & 5,19 & $\begin{array}{c}4,98 \\
(0,97)\end{array}$ & $\begin{array}{c}5,08 \\
(1,10)\end{array}$ & $\begin{array}{c}4,87 \\
(1,04)\end{array}$ & $\begin{array}{c}4,69 \\
(1,06)\end{array}$ & $\begin{array}{c}4,78 \\
(1,05)\end{array}$ & $\begin{array}{c}5,05 \\
(1,05)\end{array}$ & $\begin{array}{c}4,60 \\
(1,29)\end{array}$ & $\begin{array}{c}4,82 \\
(1,23)\end{array}$ \\
\hline $\begin{array}{l}\text { Apoyo } \\
\text { instruccional }\end{array}$ & 2,88 & 2,88 & 2,88 & 2,82 & 2,56 & 2,69 & 3,05 & 2,83 & 2,94 \\
$(0,91)$ & $(0,92)$ & $(1,05)$ & $(0,93)$ & $(0,99)$ & $(1,07)$ & $(1,39)$ & $(1,23)$ \\
\hline PROMEDIOS & 3,92 & 3,73 & 3,83 & 3,81 & 3,58 & 2,69 & 3,87 & 3,63 & 3,75 \\
$(1,90)$ & $(0,93)$ & $(0,92)$ & $(1,06)$ & $(0,98)$ & $(0,99)$ & $(1,10)$ & $(1,25)$ & $(1,18)$ \\
\hline
\end{tabular}

${ }^{* * *} p<0,01 ;{ }^{* *} p<0,05 ;{ }^{*} p<0,1$.

Como se observa, tal y como las investigaciones que utilizan CLASS indican (La Paro, Hamre, Locasale-Crouch, Pianta, Bryant, Early, Clifford y Burchinal, 2009; Stuhlman y Pianta, 2009; Treviño et al., 2013; Leyva et al., 2015), las salas analizadas poseen resultados superiores en apoyo socioemocional y organización del aula, y resultados bajos en apoyo instruccional. Con todo, es interesante notar que, en el caso específico de las actividades analizadas en el marco de esta investigación (actividades en las que hay un predominio del discurso instruccional), el dominio de organización del aula posee puntajes levemente superiores a los de apoyo socioemocional. La investigación recién mencionada sugiere que en salas de niños más pequeños (en las que se incluyen los niveles de transición), el dominio de apoyo socioemocional tiende a presentar puntajes algo superiores al de organización del aula. De este modo, estos resultados sugieren un comportamiento, al menos en algunos momentos de la jornada de los niveles de transición analizados, más parecido a lo observado para las salas de niveles superiores (Hafen, Hamre, Allen, Bell, Gitomer y Pianta, 2015): interacciones más efectivas respecto de la organización del aula y con niveles un poco inferiores para las dimensiones socioemocionales. 


\subsection{Resultados de lenguaje académico}

Respecto de los resultados de lenguaje académico, es necesario recordar que se transcribieron y analizaron las mismas experiencias observadas con el instrumento CLASS. Es decir, se trata de actividades eminentemente pedagógicas (donde la modalidad de discurso instruccional se observa la mayor parte del tiempo), en las que la educadora o la técnico poseen la palabra mayoritariamente. En específico, la actividad de rutina de la mañana (revisión del clima, del calendario, saludo y conversación) de todos los videos y otra actividad pedagógica.

En primer lugar, es importante describir someramente en términos estructurales el discurso de las educadoras. En promedio, se transcribieron 197 utterances $^{9}$ y 1.000,57 palabras por educadora, para las actividades del tiempo 1; 164,39 utterances y 877,21 palabras por educadora para las del tiempo 2; y 150,71 utterances y 814,96 palabras por educadora para el tiempo 3 .

Las actividades codificadas presentan, para el constructo "complejidad sintáctica" (MLU) un promedio de 5,503 palabras (DE $=1,912$ ). En el caso de la diversidad léxica (entregada por la medida $D)$, el promedio es de $77,53(D E=10,341)$. La presencia de palabras sofisticadas (medida como palabras sofisticadas/palabras totales o tokens) presenta un promedio de 0,5004 ( $D E=0,01624)$. Finalmente, como una medida de referencia, se da cuenta de la cantidad de palabras totales por minuto (tokens/min.) que es de 80,39 ( $D E=$ $22,895)$. En específico, para cada uno de los tiempos considerados, los descriptivos se observan en la siguiente tabla.

9 Se operacionalizó utterance como una unidad de habla que está delimitada semánticamente (una idea completa) y entonacionalmente (marcadores que dan cuenta de que una idea ha terminado). 
Tabla 3: Descriptivos de las variables de lenguaje académico para las actividades observadas en los tres tiempos considerados

\begin{tabular}{l|c|c|c|c|c|c|c|c|c|c}
\hline & \multicolumn{3}{|c|}{ Tiempo 1 } & \multicolumn{3}{c|}{ Tiempo 2 } & \multicolumn{3}{c}{ Tiempo 3 } \\
\cline { 2 - 11 } & Act. 1 & Act. 2 & Promedio & Act. 1 & Act. 2 & Promedio & Act. 1 & Act. 2 & Promedio \\
\hline Total de palabras & 90,745 & 83,111 & $86,928 * *$ & 80,995 & 77,427 & 79,211 & 78,846 & 71,217 & 75,032 \\
(tokens) / minuto & $(18,196)$ & $(23,942)$ & $(21,226)$ & $(19,113)$ & $(21,282)$ & $(19,932)$ & $(21,105)$ & $(30,859)$ & $(26,231)$ \\
\hline $\begin{array}{l}\text { Complejidad } \\
\text { sintáctica (MLU) }\end{array}$ & 5,902 & 5,372 & 5,644 & 5,643 & 5,390 & 5,517 & 5,227 & 5,464 & 5,346 \\
\hline Variedad léxica (D) & $(3,102)$ & $(2,226)$ & $(2,596)$ & $(1,536)$ & $(1,260)$ & $(1,385)$ & $(1,294)$ & $(1,885)$ & $(1,591)$ \\
\hline $\begin{array}{l}\text { Presencia de } \\
\text { vocabulario }\end{array}$ & $(35,38)$ & 78,18 & $(33,51)$ & $(33,59+27)$ & 70,27 & 75,03 & 72,65 & 79,80 & 76,62 & 78,21 \\
sofisticado & 0,0464 & 0,0539 & 0,0502 & 0,0472 & 0,0447 & 0,046 & 0,052 & 0,0555 & 0,054 \\
\hline
\end{tabular}

*** $p<0,01 ;{ }^{* *} p<0,05 ;{ }^{*} p<0,1$.

+El promedio para "Variedad léxica" es significativamente más alto para el tiempo 1 solo en relación con el tiempo 2.

Como se observa en la tabla, la cantidad de palabras por minuto para los tres tiempos no presenta una gran diferencia. De cualquier manera, en el tiempo 1 el "discurso extendido" de la educadora sería significativamente superior que en los tiempos posteriores; además, el estadístico de variedad léxica también sería superior significativamente, pero solo respecto del tiempo 2. Respecto de la presencia de vocabulario sofisticado, llama la atención su baja presencia, en general, para todas las actividades observadas: entre un $4,6 \%$ y un $5,5 \%$ promedio del total del discurso analizado para cada educadora.

En relación con este último constructo, es importante destacar algunos resultados que dan cuenta de la alta presencia de un vocabulario contrario a lo sofisticado: palabras que solo se entienden en el contexto, pues no poseen una referencia estable, sino solo dependiente de la situación en la que se utilizan. Estas palabras son, gramaticalmente, pronombres (personales, relativos, interrogativos, etc.), adverbios (ahora, así, sí, no, acá, allá) y, en específico, un verbo comodín que sirve a todo contexto: hacer. Este resultado va en el sentido inverso de lo que la literatura ha propuesto como característica léxica de este registro académico: variedad léxica y alta presencia de palabras poco frecuentes, abstractas (Schleppegrell, 2001; Snow y Uccelli, 2009; Snow, 2010; Nagy y Townsend, 2012; Dickinson et 

RELACIÓN CON EL DESARROLLO DEL LENGUAJE DE LOS NIÑOS - E. Treviño, C. Varela, F. Romo y V. Núñez

al., 2014). Asimismo, y por razones inherentes a la estructura del lenguaje, palabras como "el" (artículo), "de" o "a" (preposiciones), que sirven para relacionar otras palabras "más llenas de significado" (sustantivos, verbos, adjetivos), aparecen como las más frecuentes. En la siguiente tabla, se puede observar la frecuencia total de aparición (en la muestra completa) de algunas de estas unidades.

Tabla 4: Ejemplo de algunas de las palabras de mayor aparición en la muestra

\begin{tabular}{l|l|l}
\hline Palabra & Tipo de palabra (gramaticalmente) & $\begin{array}{l}\text { Frecuencia total / cantidad de palabras } \\
\text { total de la muestra }\end{array}$ \\
\hline A & Preposición & $3.010 / 75.397$ \\
\hline El & Artículo & $2.022 / 75.397$ \\
\hline No & Adverbio & $1.517 / 75.397$ \\
\hline Y & Conjunción, conector & $1.724 / 75.397$ \\
\hline Ya & Adverbio & $1.391 / 75.397$ \\
\hline Es & Verbo & $1.022 / 75.397$ \\
\hline Bien & Adverbio & $667 / 75.397$ \\
\hline Vamos & Verbo & $709 / 75.397$ \\
\hline Acá & Adverbio & $436 / 75.397$ \\
\hline
\end{tabular}

Como se observa, muchas de estas palabras son funcionales a la situación de la sala de clases. Por ejemplo, la palabra "vamos", se observa en las actividades cumpliendo funciones de frase verbal ("vamos a hacer...") o como regulador del ritmo de la actividad también en una frase verbal ("vamos, vamos terminando"), entre otras. El caso del conector "y" es claramente más contrario a la presencia de lenguaje académico, puesto que es más propio de este registro, el uso de marcadores más sofisticados que estructuran el discurso (como "en primer lugar", "para terminar", etc.).

Ahora bien, si los resultados generales de lenguaje académico son comparados con lo encontrado en la investigación de Dickinson et al. $(2014)^{10}$ para salas de párvulos de Estados Unidos, participantes del

10 En la investigación de estos autores, se realizó un análisis que incluyó la variable de "situación de enseñanza”, que consideraba: a) lectura de libros, b) trabajo en grupos pequeños, c) trabajo con el grupo completo en contenido. No se han comparado las medidas de presencia de palabras sofisticadas, puesto que el cálculo utilizado en Dickinson et al. (2014) es distinto del cálculo considerado en esta investigación. 
programa Head Start, lo que se observa es que, en el caso del total de palabras por minuto y de la variable de complejidad sintáctica, las salas chilenas poseen características que se asimilan a lo que sucede cuando las educadoras de Head Start trabajan en grupos pequeños; finalmente, la variable de diversidad léxica se parece a lo sucedido en momentos de lectura de libros (la comparación estadística indica, en efecto, que existe una diferencia significativa a favor de las salas analizadas en esta investigación respecto del promedio general obtenido en la investigación de Dickinson et al. (2014), y de los contextos en los que se trabaja en grupos pequeños o con el curso completo).

En síntesis, si se comparan los promedios obtenidos por las salas del Head Start con las salas chilenas, se observa que, aunque estas últimas muestran estadísticas algo menores, en el caso de la variedad léxica esto se revierte, tal como se presenta en la siguiente tabla:

Tabla 5: Comparación de las variables de lenguaje académico de las salas chilenas analizadas versus salas de Estados Unidos

\begin{tabular}{|c|c|c|c|c|c|}
\hline & \multirow{2}{*}{ 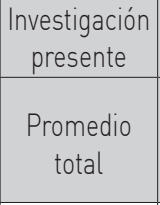 } & \multicolumn{4}{|c|}{ Dickinson et al. (2014) } \\
\hline & & $\begin{array}{l}\text { Promedio } \\
\text { total }\end{array}$ & $\begin{array}{l}\text { Lectura de } \\
\text { libros }\end{array}$ & $\begin{array}{c}\text { Trabajo } \\
\text { en grupos } \\
\text { pequeños }\end{array}$ & $\begin{array}{l}\text { Trabajo con } \\
\text { el curso en } \\
\text { contenidos }\end{array}$ \\
\hline $\begin{array}{l}\text { Total de palabras } \\
\text { (tokens) / minuto }\end{array}$ & $\begin{array}{c}80,39 \\
(22,895) \\
\end{array}$ & $\begin{array}{c}95,79 \\
(15,28)^{* * *}\end{array}$ & $\begin{array}{c}91,78 \\
(19,69)^{* *}\end{array}$ & $\begin{array}{c}86,77 \\
(24,97) \\
\end{array}$ & $\begin{array}{c}108,80 \\
(22,18)^{* * *}\end{array}$ \\
\hline $\begin{array}{l}\text { Complejidad } \\
\text { sintáctica (MLU) }\end{array}$ & $\begin{array}{c}5,503 \\
(1,912)^{+}\end{array}$ & $\begin{array}{c}6,46 \\
(1,05)^{* * *}\end{array}$ & $\begin{array}{c}8,39 \\
(2,81)^{* * *}\end{array}$ & $\begin{array}{l}4,81 \\
(0,63)\end{array}$ & $\begin{array}{c}6,20 \\
(0,96)^{* *}\end{array}$ \\
\hline Variedad léxica (D) & $\begin{array}{c}77,53 \\
(10,341)^{* * *}\end{array}$ & $\begin{array}{l}69,13 \\
(9,38)\end{array}$ & $\begin{array}{c}74,41 \\
(14,35)\end{array}$ & $\begin{array}{c}64,71 \\
(13,45)\end{array}$ & $\begin{array}{c}68,25 \\
(12,50)\end{array}$ \\
\hline
\end{tabular}

*** $p<0,01 ;{ }^{* *} p<0,05 ;{ }^{*} p<0,1$.

+ El promedio es significativamente superior al promedio obtenido por Dickinson para el contexto de trabajo en grupos pequeños.

Respecto de esta última variable, es posible que la diferencia se deba a la selección de las actividades: hay que recordar que se trata de actividades escogidas específicamente con el fin de observar mayor presencia de habla por parte de la educadora.

Análisis de regresión: los resultados de los niños y el lenguaje académico 
Antes de mostrar los resultados del análisis de regresión, es necesario revisar el comportamiento general de las variables que se consideran para realizarla. En la tabla siguiente se observan los promedios y desviación estándar de estas variables:

Tabla 6: Estadísticos descriptivos de las variables consideradas

\begin{tabular}{l|c|c|c}
\hline Variable considerada & N & Promedio & $D E$ \\
\hline WM prueba 1 & 275 & 27,28 & 5,011 \\
\hline WM prueba 3 & 262 & 12,78 & 6,100 \\
\hline WM prueba 4 & 262 & 10,14 & 1,766 \\
\hline WM prueba 7 & 288 & 4,28 & 2,496 \\
\hline Edad promedio (en meses) & 289 & 72,486 & 3,566 \\
\hline Complejidad sintáctica (MLU) (t3) & 14 & 5,346 & 1,474 \\
\hline Diversidad léxica (D) (t3) & 14 & 77,987 & 14,556 \\
\hline Presencia de vocabulario sofisticado (t3) & 14 & 0,178 & 0,035 \\
\hline CLASS (promedio total) (t3) & 14 & 4,185 & 0,625 \\
\hline
\end{tabular}

A continuación se presenta un resumen de los modelos de regresión que muestran el efecto de variables asociadas al lenguaje académico de la educadora, sobre el desarrollo del lenguaje de los niños, específicamente su nivel de vocabulario y sus habilidades de identificar letras y palabras, escritura emergente y comprensión de textos.

Con el fin de poder identificar este efecto de la manera más precisa posible, se incluyó una serie de variables que teóricamente también pueden relacionarse con el desarrollo del lenguaje de los niños en el aula. Estas variables son:

- las características de la sala (puntaje CLASS en kínder, y si la educadora del niño cambió entre prekínder y kínder);

- el rendimiento previo de los niños en las pruebas de desarrollo del lenguaje (puntaje Woodcock-Muñoz al ingresar a prekínder); y finalmente,

- el nivel educativo de la madre y algunas características del niño (género y edad).

El procedimiento para incluir todas estas variables en la estimación fue a través de la definición de una taxonomía de modelos 
de regresión, que permitió incorporarlas progresivamente. El primer modelo incluyó únicamente las variables del lenguaje académico de la educadora. El segundo modelo sumó, además, las características de la sala. El tercer modelo agregó el rendimiento previo del niño en lenguaje. Finalmente, en el último modelo se adicionó el nivel educativo de la madre, el sexo y la edad del niño. Se presume, por lo tanto, que en la incorporación sucesiva de estas variables a la estimación, el efecto del lenguaje académico sobre el desarrollo del lenguaje de los niños se identifica de forma más precisa.

En específico, las variables de lenguaje académico mostraron efectos significativos solo para las variables de resultado de identificación de letras y palabras, y de dictado. Así, en la siguiente tabla se muestran los efectos de las variables explicativas sobre estos resultados.

Tabla 7: Resumen de los efectos de las variables del lenguaje académico sobre los resultados de los niños (identificación de letras y palabras, y dictado) 
160 PRESENCIA DE LENGUAJE ACADÉMICO EN LAS EDUCADORAS DE PÁRVULOS Y SU RELACIÓN CON EL DESARROLLO DEL LENGUAJE DE LOS NIÑOS - E. Treviño, C. Varela, F. Romoy V. Núñez

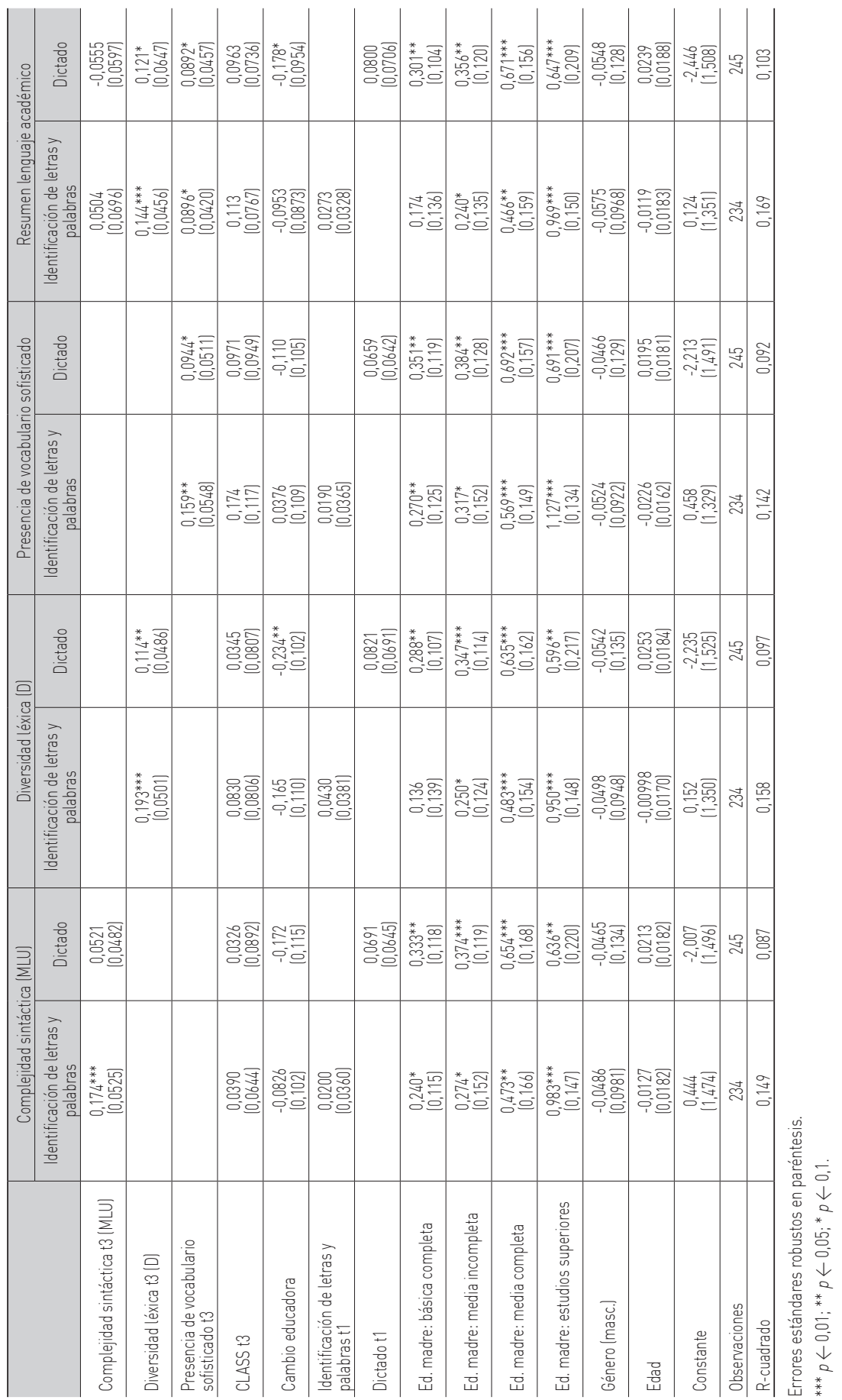


Los resultados indican que el lenguaje académico de la educadora tiene un efecto sobre las habilidades de identificación de letras y palabras, y escritura emergente de los niños (prueba de dictado en el Woodcock-Muñoz), controlando por las características de la sala, el nivel educacional de la madre y el desarrollo de lenguaje de los niños al iniciar prekínder. Los efectos más robustos ocurren sobre la capacidad de identificar letras y palabras, mientras que los efectos sobre las habilidades de escritura emergente son más débiles, pero significativos con un 90\% de confianza. A su vez, los efectos sobre estas variables tienden a mantenerse en las distintas especificaciones de los modelos de regresión, controlando o no por las características de las educadoras, las familias, y el rendimiento previo de los niños. En específico, la diversidad léxica del discurso de las educadoras muestra un efecto con mayor grado de significatividad cuando es analizado de manera independiente al resto de las variables de lenguaje académico. Lo mismo sucede con el poder explicativo del uso de palabras sofisticadas sobre la habilidad de los niños de identificar letras y palabras. De este modo, los resultados indican que, más allá de la complejidad sintáctica, los rasgos léxicos del discurso de las educadoras de párvulos predicen los resultados de los niños en habilidades relacionadas no solo con la actual comprensión oral y la futura comprensión lectora, sino sobre todo, con habilidades relacionadas directamente con la decodificación (Mckenna y Stahl, 2003).

Asimismo, el estudio de Dickinson y Porche (2011) sobre el efecto del lenguaje de la educadora en los resultados de los niños, indica que el vocabulario sofisticado utilizado por estas, afecta de manera directa sus conocimientos para la alfabetización emergente al final de kínder.

Por otra parte, también es posible explicar estos hallazgos por motivos de medición. Autores como Snow y Oh (2010) sugieren que en el campo de la evaluación del lenguaje en primera infancia, existen algunas medidas de dominio más corto que poseen propiedades psicométricas más robustas. Entre estas, se encuentra la habilidad de identificar letras y palabras y las habilidades de la decodificación en general. De las cuatro medidas del desarrollo del lenguaje infantil 
162 PRESENCIA DE LENGUAJE ACADÉMICO EN LAS EDUCADORAS DE PÁRVULOS Y SU RELACIÓN CON EL DESARROLLO DEL LENGUAJE DE LOS NIÑOS - E. Treviño, C. Varela, F. Romo y V. Núñez

incluidas en este estudio, la prueba de identificación de letras y palabras es la que más calza con este perfil.

No existen efectos estadísticamente significativos de las variables de lenguaje académico sobre el vocabulario de los niños y su nivel de comprensión de textos. Estos efectos son inexistentes prácticamente siempre, controlando o no por las características de las educadoras, de las familias y el rendimiento previo de los niños. Aunque se esperaría que sí existieran efectos, sobre todo para el caso del vocabulario de los niños (Dickinson y Porche, 2011), es necesario revisar si la cantidad de salas consideradas en este estudio no permite ver mayores efectos.

En todos los modelos, el efecto del nivel educativo de la madre sobre el desarrollo del lenguaje de los niños es consistente. Llama la atención, en este escenario, que el lenguaje académico de la educadora siga teniendo efecto positivo y estadísticamente significativo sobre el desarrollo del lenguaje de los niños. Esto quiere decir que el desarrollo del lenguaje de los niños en el nivel parvulario no solo depende del contexto educativo del hogar, sino que también lo que ocurre en la sala de clases es relevante. El tipo de lenguaje que utilice la educadora en sus interacciones con los niños va a tener un efecto independientemente del origen social de los estudiantes.

\section{Discusión y conclusiones}

El estudio realizado permitió observar algunas características del lenguaje utilizado en la sala de clases de párvulos, específicamente, en breves actividades que suceden durante la jornada de clases: el comienzo "más formal" de la jornada (el saludo) y experiencias pedagógicas variables que se basan en el trabajo de los diferentes núcleos pedagógicos explicitados en el currículo vigente de Educación de Párvulos.

Así, la investigación dio cuenta de que la calidad de las interacciones pedagógicas que se generan en estas actividades no varía, para las 14 salas observadas, en el transcurso del prekínder al kínder. Estas actividades se muestran altamente productivas, es decir, el tiempo es utilizado de forma efectiva para el desarrollo 
del aprendizaje de diferentes tipos: de habilidades, de conceptos, de rutinas, etc. Con todo, se observan pocas oportunidades para el desarrollo de habilidades superiores de pensamiento (análisis, predicciones, inferencias, etc.), aunque mayores oportunidades de desarrollo del lenguaje (uso de un lenguaje variado, de preguntas abiertas, etc.). Esto último podría estar directamente relacionado, por un lado, con la selección de las actividades en las cuales la educadora poseía un turno más extendido, y, por otro, con el hecho de ser actividades que, principalmente, revisan conceptos disciplinares (por ejemplo: enseñanza de la rima, de los cuerpos geométricos, de los diversos hábitats de animales, etc.).

Aun cuando el lenguaje presentado por las educadoras de estas salas es diverso, se observa un bajo uso de palabras sofisticadas con lo que se ven mermadas las posibilidades de conocimiento, por parte de los niños, de un vocabulario que en sus contextos cotidianos sería más difícil de encontrar. Esto último podría tener relación con la ausencia de efectos para los resultados de vocabulario en los niños, de parte de las variables de lenguaje académico de las educadoras.

En específico, los efectos encontrados sobre los resultados de identificación de letras y palabras y escritura emergente, por parte de las variables de diversidad léxica y presencia de palabras sofisticadas, da cuenta de la importancia del rol de la educadora de párvulos y de su uso de un lenguaje con unas características determinadas: al menos, la utilización de palabras distintas, y, específicamente, de palabras que los niños no tendrían oportunidad de escuchar en otros contextos. Este resultado está en línea con lo que algunas investigaciones han encontrado respecto de las características del habla del adulto en la sala de párvulos sobre sus habilidades predictoras de la lectura y escritura (Ravid y Tolchinsky, 2002; Dickinson y Porche, 2011). Al respecto, es importante decir que estas características del lenguaje de las educadoras muestran efectos robustos, incluso, al controlar por variables de contexto de los niños, como la educación de su madre. Así, la cantidad y calidad del lenguaje que utiliza la educadora de la sala, se transforma en una herramienta que podría servir para disminuir las brechas existentes entre los contextos cotidianos de los niños y lo requerido en la escuela. 
Desde la política pública, sería importante considerar en la formación inicial y continua de las educadoras de párvulos, la reflexión y enseñanza del lenguaje que les entrega a los niños mayores oportunidades para su desarrollo. En los estándares de formación de educadoras de párvulos se da cuenta, en efecto, de la importancia del uso del lenguaje por parte de estas últimas. Sin embargo, su referencia es muy amplia. Este tipo de estudios puede contribuir a entregar información más específica respecto de cuál o cuáles lenguajes se deberían enfatizar en la práctica pedagógica.

Con todo, es importante tomar algunos resguardos y no creer que la única respuesta sería la inclusión de un tipo de lenguaje como este en la formación de las educadoras. Alguna literatura, proveniente de áreas que enfatizan el rol del contexto sociocultural en las interacciones en la sala de clases, propone que la aparición de este registro depende fuertemente del contexto de comunicación. De este modo, Chafe (1986) propone la existencia de un "habla académica", que recoge aspectos lingüísticos de registros conversacionales espontáneos y cotidianos, y también aspectos de la escritura académica. Este tipo de habla ocurriría siempre que el emisor fuera conocedor experto del tema de la conversación y la audiencia (o sus interlocutores), tuvieran un conocimiento basal del mismo tema. Así, mientras mayor conocimiento de un tema, mayor es la posibilidad de utilizar un lenguaje más académico. Esto implicaría, entonces, un grado de conocimiento específico de varios temas por parte de la educadora, pero también que los niños que participan de la interacción pudieran integrar sus propios conocimientos sobre los contenidos que se están tratando.

Por otro lado, y desde una perspectiva más crítica, basada en la reflexión respecto de los orígenes y las funciones de un lenguaje de este tipo, Poza (2014) expone que cualquier posición que fije un tipo de lenguaje como una forma universal, con rasgos invariables, estará imponiendo una manera dominante de comunicación. En este contexto, la relación entre el lenguaje académico y los demás registros posibles en la sala, es de jerarquía, por medio de la concepción de que el primero sería "más puro", "apropiado" o complejo. Quienes sostienen esta posición concluyen que "(...) la adquisición de 
segundos idiomas o de variedades particulares requieren participación auténtica comunicativa y cierta socialización (...)" (Poza, 2014, p. 236). En este sentido, lo importante es dar espacio en la escuela, al intercambio y la participación en situaciones reales, auténticas, para todos los estudiantes, independientemente de su capital cultural o lingüístico. Estas oportunidades de participación deben estar enraizadas en la valoración de los diversos registros, lenguajes, conocimientos, que traen los niños y los adultos a la escuela. Así, para generar intercambios que vayan desde un lenguaje más alejado de este registro a uno que ayude a los niños en el aprendizaje de las áreas que las bases curriculares consideran relevantes, es necesario que los docentes interactúen considerando el conocimiento previo de los estudiantes y manejen una mayor cantidad y variedad de temas, que le permitirían un uso de un registro más cercano al académico.

\section{Referencias}

Bernstein, B. (1993). La estructura del discurso pedagógico: clases, código y control. Madrid: Ediciones Morata.

Bernstein, B. y Díaz, M. (1985). Hacia una teoría del discurso pedagógico. Revista Colombiana de Educación, 15, 105-153.

Chafe, W. (1986). Academic speaking. Proceedings of the twelfth annual meeting of the Berkeley Linguistics Society: General session and parasession on semantic typology. Berkeley, California: Berkeley Linguistics Society.

Christie, F. \& Martin, J. (2007). Language, knowledge and pedagogy: Functional linguistic and sociological perspectives. New York: Continuum.

Concha, S., Treviño, E., Romo, F., Varela, C., Arratia, A., Garrido, M., y Arias, R. (2010). Evaluación de estrategias de desarrollo profesional LEM y ECBI. Santiago de Chile: Universidad Diego Portales.

Dickinson, D. \& Porche, M. (2011). Relation between language experiences in preschool classrooms and children's kindergarten and fourth-grade language and reading abilities. Child development, 82(3), 870-886. http://dx.doi:10.1111/j.1467-624.2011.01576.x

Dickinson, D., Hofer, K., Barnes, E., \& Grifenhagen, J. (2014). Examining teachers' language in Head Start classrooms from a Systemic Linguistics Approach. Early Childhood Research Quarterly, 29(3), 231-244. http:// dx.doi:10.1016/j.ecresq.2014.02.006 
166 PRESENCIA DE LENGUAJE ACADÉMICO EN LAS EDUCADORAS DE PÁRVULOS Y SU RELACIÓN CON EL DESARROLLO DEL LENGUAJE DE LOS NIÑOS - E. Treviño, C. Varela, F. Romo y V. Núñez

Durán, P., Malvern, D., Richards, B., \& Chipere, N. (2004). Developmental trends in lexical diversity. Applied Linguistics, 25(2), 220-242. http:// dx.doi:10.1093/Applin/25.2.220

Hafen, C., Hamre, B., Allen, J., Bell, C., Gitomer, D., \& Pianta, R. (2015). Teaching through interactions in secondary school classrooms: Revisiting the factor structure and practical application of the classroom assessment scoring system-secondary. Journal of Early Adolescence, 35(5-6), 651-680. http://dx.doi:10.1177/0272431614537117

Halliday, M. (1979). El lenguaje como semiótica social. México: Fondo de Cultura Económica.

Halliday, M. (1993). Towards a language-based theory of learning. Linguistics and Education, 5, 93-116. http://dx.doi 10.5040/9781474212007. ch-015

Harper-Browne, C. \& Raikes, H. (2012). Essential elements of quality infant-toddler programs. Minnesota Department of Education and the Center for Early Education and Development, University of Minnesota. Recuperado de http://www.cehd.umn.edu/ceed/projects/ essentialelements/EssentialElementsOfInfantsAndToddlersReportN ov2012.pdf

Hicks, D. (Ed.). (1996). Discourse, learning, and schooling. Cambridge, UK: Cambridge University Press.

Huttenlocher, J., Vasilyeva, M., Cymerman, E., \& Levine, S. (2002). Language input and child syntax. Cognitive Psychology, 45, 337-374.

La Paro, K., Hamre, B., Locasale-Crouch, J., Pianta, R., Bryant, D., Early, D., Clifford, R., Burchinal, M. (2009). Quality in kindergarten classrooms: Observational evidence for the need to increase children's learning opportunities in early education classrooms. Early Education \& Development, 20(4), 657-692. http://dx.d oi:10.1080/10409280802541965

Lagos, C., Treviño, E., Concha, S., Romo, F., y Varela, C. (2010). Informe final: evaluación de efectividad del Proyecto LEAMOS. Santiago de Chile: Universidad Diego Portales.

Leyva, D., Weiland, C., Barata, M., Yoshikawa, H., Snow, C., Treviño, E., $\&$ Rolla, A. (2015). Teacher-child interactions in Chile and their associations with prekindergarten outcomes. Child development, 86(3), 781-799. http://dx.doi:10.1111/cdev.12342.

MacWhinney, B. (2000). The CHILDES Project: Tools for analyzing talk-electronic edition. Mahwah, NJ: Lawrence Erlbaum Associates. Recuperado de http://childes.talkbank.org/manuals/chat.pdf 
Martin, J. (2013). Embedded literacy: Knowledge as meaning. Linguistics and Education, 24(1), 23-37. http://dx.doi:10.1016/j.linged.2012.11.006

Mckenna, M. \& Stahl, S. (2003). Assessment for reading instruction. New York: The Guilford Press.

Ministerio de Educación de Chile, Mineduc. (2003). Programa de estudio cuarto año básico. Lenguaje y Comunicación. Santiago de Chile: Autor.

Moyano, E. (2013). Aprender ciencias y humanidades: una cuestión de lectura y escritura. Aportes para la construcción de un programa de inclusión social a través de la educación lingüística. Buenos Aires: Universidad Nacional de General Sarmiento.

Nagy, W. \& Townsend, D. (2012). Words as tools: Learning academic vocabulary as language acquisition. Reading Research Quarterly, 47(1), 91-108. http://dx.doi:10.1002/RRQ.011

Pianta, R., LaParo, K., \& Hamre, B. (2008). Classroom Assessment Scoring System $^{\mathrm{TM}}\left(\right.$ CLASS $\left.^{\mathrm{TM}}\right)$. Baltimore, Maryland: Paul H. Brookes Publishing Co., Inc.

Poza, L. (2014). Mecanismos de exclusión: ideologías lingüísticas y ambigüedades en la conceptualización de lenguaje académico. Miriada Hispánica, 10, 225-244.

Ravid, D. \& Tolchinsky, L. (2002). Developing linguistic literacy: A comprehensive model. Journal of Child Language, 29(2), 417-447. http:// dx.doi.org/10.1017/S0305000902005111

Schleppegrell, M. J. (2001). Linguistic features of the language of schooling. Linguistics and Education 12(4), 431-459. http://dx.doi:10.1016/S08985898(01)00073-0

Schleppegrell, M. (2012). Academic language in teaching and learning introduction to the special issue. The Elementary School Journal, 112(3), 409-418. http://dx.doi:10.1086/663297

Snow, C. (2010). Academic language and the challenge of reading for learning about science. Science, 328(5977), 450-452. http://dx.doi:10.1126/ science. 1182597

Snow, C. \& Ucceli, P. (2009). The challenge of academic language. En D. Olson \& N. Torrance (Eds.), The Cambridge Handbook of Literacy (pp. 112-133). Cambridge, UK: Cambridge University Press.

Snow, C. E. \& Oh, S. S. (2010). Assessment in early literacy research. En S. B. Neuman \& D. K. Dickinson (Eds.), Handbook of Early Literacy Research (Vol. 3, pp. 375-395). New York: Guilford Press. 
Stuhlman, M. \& Pianta, R. (2009). Profiles of educational quality in first grade. The Elementary School Journal, 109(4), 323-342. http:// dx.doi:10.1086/593936

Treviño, E., Toledo, G., y Gempp, R. (2013). Calidad de educación parvularia: las prácticas de clase y el camino a la mejora. Pensamiento Educativo. Revista de Investigación Educacional Latinoamericana, 50(1), 40-62. http://dx.doi.org/10.7764/PEL.50.1.2013.4

Vygotsky, L. (1934/2002). Pensamiento y lenguaje. España: Paidós.

Woodcock, R., Muñoz, A., Ruef, M., \& Alvarado, C. (2005). Woodcock-Muñoz Language Survey ${ }^{\circledR}$ Revised. Itasca, IL: Riverside Publishing.

Yoshikawa, H., Leyva, D., Snow, C. E., Treviño, E., Barata, M., Weiland, C., \& Arbour, M. C. (2015). Experimental impacts of a teacher professional development program in Chile on preschool classroom quality and child outcomes. Developmental psychology, 51(3), 309-322. http:// dx.doi.org/10.1037/a0038785

Recibido: 06/11/2015

Aceptado: 07/12/2015 